\title{
Physical Education and Leisure-Time Physical Activity in Youth Are Both Important for Adulthood Activity, Physical Performance, and Health
}

\author{
Elin Ekblom-Bak, Örjan Ekblom, Gunnar Andersson, Peter Wallin, and Björn Ekblom
}

\begin{abstract}
Background: The importance of youth physical activity (PA) for adulthood PA, performance, and health was retrospectively evaluated. Methods: A total of 258,146 participants (49\% women), aged 19-70, with a first-time health-profile assessment between 1982 and 2015, provided self-reported data on current perceived health, PA, lifestyle, and physical education class participation, and PA outside school hours before age 20. Data on anthropometrics, blood pressure, and estimated maximal oxygen consumption $\left(\mathrm{VO}_{2 \max }\right)$ were obtained. Results: Women participating in physical education class, compared with those who did not, had significantly lower OR (range: 0.81-0.87) for perceiving poor overall health, general obesity, and high diastolic blood pressure after adjustment for potential confounders, and increased OR (range: 1.17-1.23) for exercising regularly and a normal/high $\mathrm{VO}_{2 \max }$ in adulthood. For men, the ORs were significantly lower (range: 0.66-0.86) for poor perceived overall health, general, and abdominal obesity. These associations were seen for participants up to 70 years. Increased PA outside school hours revealed even stronger beneficial associations. In joint analyses, both youth and current PA were important for lower OR of poor health and being obese in adulthood. Conclusions: Physical education class participation and additional PA after school hours were both important for perceived health, $\mathrm{PA}, \mathrm{VO}_{2 \max }$, and metabolic health in adulthood up to 70 years.
\end{abstract}

Keywords: adolescent, epidemiology, physical fitness, public health, $\mathrm{VO}_{2 \max }$

Despite the well-established knowledge that physical activity (PA) is important for health and longevity, the majority of the adult population does not meet current national guidelines. ${ }^{1,2}$ Even if current PA level is known to have the greatest effect on health and physical performance in general,, 3 childhood and adolescence PA might also have important beneficial effects in different domains, for example, bone density. ${ }^{5}$ With the alarming reports of increasing childhood/adolescent obesity and declining fitness levels, ${ }^{6,7}$ studies on the immediate effects on health and disease in youth, as well as tracking into adulthood and effects on lifestyle, activity habits, and health later in life, are highly relevant. Previous tracking studies have reported, in general, low to moderate correlation between childhood and adult PA and fitness, with some equivocal results between different domains of youth PA. ${ }^{8-11}$ This might be due to methodological issues as well as small sample sizes. Follow-ups are mainly performed over the early parts of the adult life span, up to 26-39 years, $, 9,10,12,13$ with limited long-term follow-ups from childhood to late adulthood. In addition, little is known about the effects of youth PA and cardiovascular risk later in life. ${ }^{14}$ Moreover, although physical education (PE) class at least once a week has been and is provided to all Swedish children/adolescents from first grade (7 y) until leaving high school at the age of 16, there is a paucity of research that specifically studies the importance of school-based PE class participation.

Health profile assessment (HPA) is an interdisciplinary method combining medicine, physiology, and behavioral science to promote health. This method has been used in occupational health services in Sweden for almost 40 years, with several thousand participants

Ekblom-Bak, Ö. Ekblom, and B. Ekblom are with the Åstrand Laboratory of Work Physiology, The Swedish School of Sport and Health Sciences, Stockholm, Sweden. Andersson and Wallin are with the Research Dept, HPI Health Profile Institute AB, Stockholm, Sweden. Ekblom-Bak (eline@gih.se) is corresponding author. carrying out an HPA every year in different places around Sweden. It is composed of a person-centered dialogue and includes selfreported lifestyle and perceived health, previous and current PA habits, and metabolic and physiological measurements. All data are subsequently registered and stored in a database. The combination of the large amount of HPA performed each year and the long-term use of well-established and standardized methods in occupational health promotion generates a unique database, which enables analyses of youth PA habits in relation to adulthood PA habits, performance, and health in the Swedish working population. The large amount of data also enables sex- and age-stratified analyses.

The primary aim of this study was to retrospectively analyze the importance of PA before 20 years of age, specified as participation in PE class or not, together with additional PA outside school hours, on adult PA level, maximal oxygen consumption $\left(\mathrm{VO}_{2 \max }\right)$, and perceived and metabolic health later in life in a large sample of Swedish men and women of a broad age span.

\section{Methods}

From October 1982 to September 2015, 363,746 men and women, aged 19-70, performed a first-time HPA, which was registered and stored in the database. HPI Health Profile Institute AB (Stockholm, Sweden) is the institute that is responsible for the database, standardization of methods used, and education of the HPA coaches since the start of the administration of HPAs in the late 1970s. In October 2015, data on participants were obtained from the database and used for the present analyses. The protocols used were approved by the institutional review board of HPI Health Profile Institute $\mathrm{AB}$, and all participants provided informed consent prior to data collection. The study was approved by the ethics board at Karolinska University (Dnr 2015/1864-31/2) and adhered to the Declaration of Helsinki. 


\section{Health Profile Assessment}

The HPA method comprises 3 components, which have been scientifically evaluated ${ }^{15,16}$ : current perceived health, self-assessment of lifestyle habits, and some medical and physiological measurements. Participation is optional and free of charge for the individual and is offered to all employees working for a company or organization connected to occupational or other health service. The participant answers an extensive questionnaire including current lifestyle, PA habits, and perceived overall stress and health. During a following dialogue with an HPA coach, the participant provides information regarding age, marital status, occupation, and PA level prior to the age of 20, with the latter including participation or not in PE class and additional PA outside school hours. Subsequently, weight, height, waist circumference, and blood pressure (BP) data are obtained. Finally, the participant performs a submaximal exercise test on a cycle ergometer for estimation of $\mathrm{VO}_{2 \max }$. All data are recorded in the HPI Health Profile Institute AB database.

\section{PA Habits}

PA level prior to the age of 20 was self-reported by selecting one of the following 5 given alternatives through the statement: Prior to the age of 20, I . . Did not participate in physical education (PE) class, Participated only in PE class, Participated in PE class + 1-2 times/week of PA outside school hours, Participated in PE class + 3-5 times/week of PA outside school hours, or Participated in PE class + At least 6 times/week of PA outside school hours. Current exercise and commuting habits were self-reported through the statement: I exercise for the purpose of maintaining/improving my physical fitness, health and well-being . . . with the alternatives Never, Sometimes, 1-2 times/week, 3-5 times/week, or At least 6 times/week. For current commuting, the statement I walk or cycle to and/or from work. . . was used with the alternatives Less than $5 \mathrm{~min} /$ day, 5-9 min/day, 10-19 min/day, 20-29 min/day, or At least $30 \mathrm{~min} / \mathrm{day}$.

\section{Perceived Health and Covariates}

Data on perceived overall health and overall stress, as well as on diet, smoking, and alcohol habits were obtained from the questionnaire responses. Perceived overall health was assessed using the statement: I perceive my physical and mental health as . . . with the alternatives Very poor, Poor, Neither good or bad, Good, or Very good. Perceived overall stress was assessed using the statement I perceive stress in my life, both personally and at work. . . with the alternatives Very often, Often, Sometimes, Rarely, or Never. Diet habits were obtained using the statement I consider my diet, regarding both meal frequency and nutritional content to be ... with the alternatives Very poor, Poor, Neither good nor bad, Good, or Very good. Smoking habits were obtained using the statement I smoke. . . with the alternatives At least 20 cig/day, 11-19 cig/day, 1-10 cig/day, Occasionally, or Never. The statement regarding alcohol use since the year 1982 was I drink alcohol ... with the alternatives Very often, Often, Sometimes, Rarely, or Never, but this was changed in 2011 to I consider my alcohol habits, from a health perspective, to be . . . with the alternatives Very poor, Poor, Neither good nor bad, Good, or Very good. Although the alcohol use statement and alternatives have been changed throughout the years, the variable is kept in the analyses as a proxy for alcohol use as the answer alternatives in both occasions are on a 5-point ordinal scale, and the variable is only used as a covariate in the analyses with small variation between the different subgroups of the main exposure (PA) prior to the age of 20 (see Tables 1 and 2).

In the dialogue, marital status was reported as either Living together, Living together with children, Living alone, or Living alone with children. Occupation was reported according to the Swedish Standard Classification of Occupations 1996 (SSYK96) until June 2014 and according to the SSYK 2012 after that. SSYK is a system for classifying and aggregating data about occupations in administrative registers or statistical surveys. Occupations reported according to both SSYK96 and SSYK 2012 can be further grouped into 4 broad skill levels defined by the level of education for the particular occupation; level 1 covers elementary education at the primary school level, meaning no or a low formal education requirements; level 2 covers education programs at the upper secondary and tertiary level of no more than 2 years in length; level 3 covers practical or vocational tertiary education programs of 2-3 years in length; and level 4 covers theoretical or researchoriented tertiary education programs and third-cycle programs of at least 3 years, normally 4 years or longer in length.

\section{Measured Health and Performance}

Body mass was obtained with a calibrated scale in lightweight clothing to the nearest $0.5 \mathrm{~kg}$. Body height was measured to the nearest $0.5 \mathrm{~cm}$ using a stadiometer. Body mass index (BMI) was computed as body mass divided by body height in meters squared $\left(\mathrm{kg} / \mathrm{m}^{2}\right)$. Waist circumference was measured with a tape measure to the nearest $0.5 \mathrm{~cm}$ at the midpoint between the top of the iliac crest and the lower margin of the last palpable rib in the midaxillary line after normal exhalation. Systolic and diastolic BPs ( $\mathrm{mm} \mathrm{Hg}$ ) were measured manually in the right arm using the standard auscultatory method after 20 minutes of seated resting. $\mathrm{VO}_{2 \max }$, expressed as $\mathrm{mL} / \mathrm{min} / \mathrm{kg}$, was estimated from heart rate response after 6-minute submaximal exercise on a cycle ergometer according to Åstrand. ${ }^{17}$

\section{Statistical Analysis}

Continuous data displayed nonnormality according to the Kolmogorov-Smirnov test and were summarized as medians with quartile 1 and quartile 3 in Tables 1 and 2. Significant differences between the levels of PA prior to age 20 for the continuous data were tested for by Kruskal-Wallis analysis of variance with pairwise comparison (adjusting for multiple comparisons). The nominal and ordinal data obtained during the dialogue and through questionnaire responses were further dichotomized according to the definition in Tables 1 and 2. Significant differences between the levels of PA prior to age 20 for the proportions were tested by comparing proportions with the $99 \%$ confidence interval to compensate for multiple testing. In Tables 3-5, logistic regression models were used to assess the odds ratio (OR) and 95\% confidence interval associated with higher levels of PA prior to the age of 20 for the dichotomized variables of overall health, current exercise habits, and intake of heart medicine (as a proxy of underlying disease). $\mathrm{VO}_{2 \max }$, $\mathrm{BMI}$, waist circumference, and systolic and diastolic BPs were all dichotomized according to conventional cutoff points for increased health risks $\left(\mathrm{VO}_{2 \max }<32 \mathrm{~mL} /\right.$ $\mathrm{min} / \mathrm{kg}$ in women and $<35$ in men; BMI $\geq 30 \mathrm{~kg} / \mathrm{m}^{2}$; waist circumference $\geq 88 \mathrm{~cm}$ in women and $\geq 102 \mathrm{~cm}$ in men; systolic $\mathrm{BP} \geq$ $140 \mathrm{~mm} \mathrm{Hg}$; diastolic BP $\geq 90 \mathrm{~mm} \mathrm{Hg}$ ). Nagelkerke's $R^{2}$ was reported as regression model fit diagnostics. All analyses were performed using IBM SPSS (version 21.0; SPSS Inc, Chicago, IL) and Confidence Interval Analysis (version 2.2.0, (C) Trevor N Bryant in 2000). 
Table 1 Characteristics of Women in the Study Population $(\mathrm{N}=126,438)$

\begin{tabular}{|c|c|c|c|c|c|}
\hline & $\begin{array}{c}\text { No PE class, } \\
n=1793\end{array}$ & $\begin{array}{c}\text { Only PE class, } \\
n=32,449\end{array}$ & $\begin{array}{c}\text { PE class }+ \text { PA } \\
1-2 \text { times/wk, } \\
n=51,680\end{array}$ & $\begin{array}{c}\text { PE class }+ \text { PA } \\
3-5 \text { times/wk, } \\
n=34,134\end{array}$ & $\begin{array}{c}\text { PE class + PA } \\
\geq 6 \text { times/wk, } \\
n=6382\end{array}$ \\
\hline Age, y & $47(37-54)^{a, b, c, d}$ & $49(40-56)^{\mathrm{b}, \mathrm{c}, \mathrm{d}}$ & $45(36-54)^{\mathrm{c}, \mathrm{d}}$ & $40(33-49)^{\mathrm{d}}$ & $39(31-47)$ \\
\hline Relationship (live together) & $72 \%{ }^{\mathrm{a}, \mathrm{b}, \mathrm{c}, \mathrm{d}}$ & $77 \%$ & $77 \%$ & $77 \%$ & $76 \%$ \\
\hline Level of education (level 4) & $22 \%$ b,c,d & $22 \% \%^{\mathrm{b}, \mathrm{c}, \mathrm{d}}$ & $29 \% \%^{\mathrm{c}, \mathrm{d}}$ & $32 \%{ }^{\mathrm{d}}$ & $38 \%$ \\
\hline Overall health (very poor/poor) & $11 \%^{\mathrm{a}, \mathrm{b}, \mathrm{c}, \mathrm{d}}$ & $8 \%^{\mathrm{b}, \mathrm{c}, \mathrm{d}}$ & $6 \%$ & $6 \%$ & $6 \%$ \\
\hline Overall stress (very often/often) & $23 \% \%^{\mathrm{a}, \mathrm{b}, \mathrm{c}, \mathrm{d}}$ & $19 \%$ & $19 \%$ & $19 \%$ & $18 \%$ \\
\hline Diet habits (very poor/poor) & $9 \%$ a,b,c,d & $6 \%{ }^{\mathrm{b}, \mathrm{c}, \mathrm{d}}$ & $5 \%$ & $5 \%$ & $4 \%$ \\
\hline Smoking habits $(\geq 1 \mathrm{cig} / \mathrm{d})$ & $19 \% \%^{\mathrm{a}, \mathrm{b}, \mathrm{c}, \mathrm{d}}$ & $15 \%$ b,c,d & $13 \%{ }^{\mathrm{c}, \mathrm{d}}$ & $12 \%{ }^{\mathrm{d}}$ & $10 \%$ \\
\hline Alcohol habits (very poor/poor) & $4 \%$ & $3 \%$ & $3 \%$ & $3 \%$ & $3 \%$ \\
\hline Current exercise ( $\geq 1$ time/wk) & $58 \%^{\mathrm{a}, \mathrm{b}, \mathrm{c}, \mathrm{d}}$ & $63 \% \%^{\mathrm{b}, \mathrm{c}, \mathrm{d}}$ & $71 \%{ }^{\mathrm{c}, \mathrm{d}}$ & $74 \%^{\mathrm{d}}$ & $78 \%$ \\
\hline Current commuting ( $\geq 10 \mathrm{~min} / \mathrm{d}$ ) & $30 \%$ & $29 \%$ b,d & $31 \%$ & $30 \%{ }^{\mathrm{d}}$ & $32 \%$ \\
\hline $\mathrm{VO}_{2 \max }, \mathrm{mL} / \mathrm{min} / \mathrm{kg}$ & $32(26-38)^{\mathrm{b}, \mathrm{c}, \mathrm{d}}$ & $32(27-38)^{\mathrm{b}, \mathrm{c,d}}$ & $34(29-41)^{\mathrm{c}, \mathrm{d}}$ & $37(31-44)^{\mathrm{d}}$ & $39(32-47)$ \\
\hline BMI, $\mathrm{kg} / \mathrm{m}^{2}$ & $25.1(22.5-28.7)^{\mathrm{a}, \mathrm{b}, \mathrm{c}, \mathrm{d}}$ & $24.8(22.4-27.9)^{\mathrm{b}, \mathrm{c}, \mathrm{d}}$ & $24.2(22.0-27.2)^{\mathrm{c}, \mathrm{d}}$ & $23.9(21.7-26.7)^{\mathrm{d}}$ & $23.6(21.5-26.4)$ \\
\hline Waist circumference, $\mathrm{cm}$ & $88.0(79.0-99.3)^{\mathrm{b}, \mathrm{c}, \mathrm{d}}$ & $86.0(78.5-95.0)^{\mathrm{b}, \mathrm{c}, \mathrm{d}}$ & $84.0(77.0-92.0)^{\mathrm{c}, \mathrm{d}}$ & $82.0(76.0-91.0)$ & $81.0(75.0-91.0)$ \\
\hline Systolic BP, mm Hg & $123(112-135)^{\mathrm{b}, c, \mathrm{~d}}$ & $125(115-136)^{\mathrm{b}, \mathrm{c}, \mathrm{d}}$ & $120(110-132)^{\mathrm{c}, \mathrm{d}}$ & $120(110-130)^{\mathrm{d}}$ & $120(110-129)$ \\
\hline Diastolic BP, mm Hg & $79(70-85)^{\mathrm{b}, \mathrm{c}, \mathrm{d}}$ & $80(70-85)^{\mathrm{b}, \mathrm{c}, \mathrm{d}}$ & $76(70-82)^{\mathrm{c}, \mathrm{d}}$ & $75(70-80)^{\mathrm{d}}$ & $75(70-80)$ \\
\hline Taking heart medicine (yes) & $8 \%^{\mathrm{b}, \mathrm{c}, \mathrm{d}}$ & $8 \%^{\mathrm{b}, \mathrm{c}, \mathrm{d}}$ & $6 \%$ c,d & $4 \%$ & $4 \%$ \\
\hline
\end{tabular}

Note. Data are presented as median $(\mathrm{Q} 1-\mathrm{Q} 3)$ or $\%$.

Abbreviations: BMI, body mass index; BP, blood pressure; cig, cigarette; PA, physical activity; PE, physical education; $\mathrm{VO}_{2 \text { max }}$, maximal oxygen consumption.

${ }^{\text {a }}$ Significant difference versus only PE class.

${ }^{\mathrm{b}}$ Significant difference versus PE class + PA 1-2 times/week.

${ }^{c}$ Significant difference versus PE class + PA 3-5 times/week.

${ }^{\mathrm{d}}$ Significant difference versus PE class $+\mathrm{PA} \geq 6$ times/week.

'The question was changed in 2011, see the "Methods" section.

Table 2 Characteristics of Men in the Study Population $(\mathrm{N}=131,708)$

\begin{tabular}{|c|c|c|c|c|c|}
\hline & $\begin{array}{c}\text { No PE class, } \\
n=1289\end{array}$ & $\begin{array}{c}\text { Only PE class, } \\
n=18,628\end{array}$ & $\begin{array}{c}\text { PE class + PA } \\
1-2 \text { times/wk, } \\
n=39,925\end{array}$ & $\begin{array}{c}\text { PE class + PA } \\
3-5 \text { times/wk, } \\
n=56,491\end{array}$ & $\begin{array}{c}\text { PE class + PA } \\
\geq 6 \text { times/wk } \\
n=15,375\end{array}$ \\
\hline Age, y & $44(34-53)^{\mathrm{a}, \mathrm{b}, \mathrm{c}}$ & $46(36-54)^{\mathrm{b}, \mathrm{c}, \mathrm{d}}$ & $44(35-53)^{\mathrm{b}, \mathrm{c}}$ & $42(33-51)^{\mathrm{c}}$ & $40(32-48)$ \\
\hline Relationship (live together) & $72 \%{ }^{\mathrm{c}, \mathrm{d}}$ & $71 \%{ }^{\mathrm{b}, \mathrm{c}, \mathrm{d}}$ & $74 \%{ }^{\mathrm{c}, \mathrm{d}}$ & $77 \%^{\mathrm{d}}$ & $79 \%$ \\
\hline Level of education (level 4) & $24 \% \%^{\mathrm{b}, \mathrm{c}, \mathrm{d}}$ & $27 \%^{\mathrm{b}, \mathrm{c}, \mathrm{d}}$ & $30 \%^{\mathrm{d}}$ & $30 \%{ }^{\mathrm{d}}$ & $35 \%$ \\
\hline Overall health (very poor/poor) & $10 \%^{\mathrm{a}, \mathrm{b}, \mathrm{c}, \mathrm{d}}$ & $6 \%{ }^{\mathrm{b}, \mathrm{c}, \mathrm{d}}$ & $6 \%$ c,d & $5 \%$ & $5 \%$ \\
\hline Overall stress (very often/often) & $11 \%$ & $11 \%{ }^{\mathrm{c}}$ & $10 \%^{\mathrm{c}}$ & $10 \%{ }^{\mathrm{d}}$ & $11 \%$ \\
\hline Diet habits (very poor/poor) & $15 \%{ }^{\mathrm{a}, \mathrm{b}, \mathrm{c}, \mathrm{d}}$ & $15 \%^{\mathrm{b}, \mathrm{c}, \mathrm{d}}$ & $11 \%{ }^{\mathrm{c}, \mathrm{d}}$ & $10 \%{ }^{\mathrm{d}}$ & $9 \%$ \\
\hline Smoking habits ( $\geq 1 \mathrm{cig} / \mathrm{d})$ & $18 \%^{\mathrm{a}, \mathrm{b}, \mathrm{c}, \mathrm{d}}$ & $13 \%^{\mathrm{b}, \mathrm{c}, \mathrm{d}}$ & $11 \%{ }^{\mathrm{c}, \mathrm{d}}$ & $9 \%^{\mathrm{d}}$ & $7 \%$ \\
\hline Alcohol habits (very poor/poor) ${ }^{\mathrm{e}}$ & $8 \% \%^{\mathrm{a}, \mathrm{b}, \mathrm{c}, \mathrm{d}}$ & $7 \% \mathrm{c}, \mathrm{d}$ & $6 \%$ & $6 \%$ & $6 \%$ \\
\hline Current exercise ( $\geq 1$ time/wk) & $46 \%^{\mathrm{b}, \mathrm{c}, \mathrm{d}}$ & $46 \%^{\mathrm{b}, \mathrm{c}, \mathrm{d}}$ & $56 \% \%^{\mathrm{c}, \mathrm{d}}$ & $65 \%{ }^{\mathrm{d}}$ & $72 \%$ \\
\hline Current commuting $(\geq 10 \mathrm{~min} / \mathrm{d})$ & $21 \%$ & $22 \%$ & $23 \%$ & $22 \%$ & $23 \%$ \\
\hline $\mathrm{VO}_{2 \max }, \mathrm{mL} / \mathrm{min} / \mathrm{kg}$ & $33(27-40)^{\mathrm{b}, \mathrm{c}, \mathrm{d}}$ & $33(28-39)^{\mathrm{b}, \mathrm{c}, \mathrm{d}}$ & $35(29-41)^{\mathrm{b}, \mathrm{c}}$ & $37(30-44)^{\mathrm{c}}$ & $39(32-47)$ \\
\hline BMI, $\mathrm{kg} / \mathrm{m}^{2}$ & $26.1(23.9-29.0)^{\mathrm{b}, \mathrm{d}}$ & $25.9(23.7-28.6)^{\mathrm{b}, \mathrm{d}}$ & $25.7(23.6-28.2)^{\mathrm{b}, \mathrm{c}}$ & $25.8(23.8-28.1)^{\mathrm{c}}$ & $25.9(23.9-28.2)$ \\
\hline Waist circumference, $\mathrm{cm}$ & $98.0(90.8-107.0)^{\mathrm{b}, \mathrm{c}, \mathrm{d}}$ & $97.0(90.0-105.0)^{\mathrm{b}, \mathrm{c}, \mathrm{d}}$ & $96.0(89.0-103.0)^{\mathrm{b}, \mathrm{c}}$ & $94.0(88.0-102.0)$ & $94.0(88.0-101.0)$ \\
\hline Systolic BP, mm Hg & $130(120-140)^{\mathrm{b}, \mathrm{c}, \mathrm{d}}$ & $130(120-140)^{\mathrm{b}, \mathrm{c}, \mathrm{d}}$ & $130(120-139)^{\mathrm{b}, \mathrm{c}}$ & $126(120-136)^{\mathrm{c}}$ & $125(120-135)$ \\
\hline Diastolic BP, mm Hg & $80(70-87)^{\mathrm{b}, \mathrm{d}}$ & $80(74-85)^{\mathrm{b}, \mathrm{c}, \mathrm{d}}$ & $80(70-85)^{\mathrm{b}, \mathrm{d}}$ & $80(70-85)^{\mathrm{d}}$ & $80(70-85)$ \\
\hline Taking heart medicine (yes) & $9 \%^{\mathrm{b}, \mathrm{c}, \mathrm{d}}$ & $8 \%^{\mathrm{b}, \mathrm{c}, \mathrm{d}}$ & $7 \%^{\mathrm{b}, \mathrm{c}}$ & $6 \%^{\mathrm{c}}$ & $5 \%$ \\
\hline
\end{tabular}

Note. Data are presented as median $(\mathrm{Q} 1-\mathrm{Q} 3)$ or $\%$.

Abbreviations: BMI, body mass index; $\mathrm{BP}$, blood pressure; cig, cigarette; PA, physical activity; PE, physical education; $\mathrm{VO}_{2 \max }$, maximal oxygen consumption.

${ }^{a}$ Significant difference versus only PE class.

${ }^{\mathrm{b}}$ Significant difference versus PE class + PA 3-5 times/week.

${ }^{\mathrm{c}}$ Significant difference versus $\mathrm{PE}$ class $+\mathrm{PA} \geq 6$ times/week.

${ }^{\mathrm{d}}$ Significant difference versus PE class + PA 1-2 times/week.

eThe question was changed in 2011, see the "Methods" section. 
Table 3 OR $(95 \% \mathrm{Cl})$ for Different Levels of PA Prior to the Age of 20 in Relation to Dichotomized Variables of Perceived Health, Exercise Habits, $\mathrm{VO}_{2 \max }$, and Metabolic Health in Women

\begin{tabular}{|c|c|c|c|c|c|c|}
\hline & $\begin{array}{l}\text { No PE } \\
\text { class }\end{array}$ & Only PE class & $\begin{array}{c}\text { PE class + PA } \\
1-2 \text { times/wk }\end{array}$ & $\begin{array}{l}\text { PE class + PA } \\
3-5 \text { times/wk }\end{array}$ & $\begin{array}{c}\text { PE class }+\mathrm{PA} \\
\geq 6 \text { times/wk }\end{array}$ & $R^{2}$ \\
\hline \multicolumn{7}{|c|}{ Overall health (very poor/poor) } \\
\hline Age adjusted & 1 & $0.68(0.58-0.79)$ & $0.57(0.49-0.66)$ & $0.53(0.45-0.62)$ & $0.53(0.44-0.63)$ & .002 \\
\hline Lifestyle adjusted & 1 & $0.84(0.71-0.99)$ & $0.81(0.68-0.96)$ & $0.81(0.69-0.97)$ & $0.89(0.73-1.09)$ & .19 \\
\hline \multicolumn{7}{|c|}{ Current exercise ( $\geq 1$ time/wk) } \\
\hline Age adjusted & 1 & $1.27(1.16-1.40)$ & $1.82(1.65-2.00)$ & $2.21(2.00-2.43)$ & $2.71(2.42-3.02)$ & .02 \\
\hline Lifestyle adjusted & 1 & $1.17(1.06-1.30)$ & $1.58(1.42-1.74)$ & $1.87(1.69-2.07)$ & $2.21(1.97-2.49)$ & .11 \\
\hline \multicolumn{7}{|c|}{ Normal/high $\mathrm{VO}_{2 \max }(\geq 32 \mathrm{~mL} / \mathrm{min} / \mathrm{kg})$} \\
\hline Age adjusted & 1 & $1.26(1.12-1.41)$ & $1.72(1.53-1.94)$ & $2.13(1.89-2.40)$ & $2.52(2.20-2.88)$ & .19 \\
\hline Lifestyle adjusted & 1 & $1.23(1.09-1.39)$ & $1.50(1.33-1.70)$ & $1.78(1.58-2.01)$ & $1.98(1.83-2.27)$ & .26 \\
\hline \multicolumn{7}{|l|}{ BMI $\left(\geq 30 \mathrm{~kg} / \mathrm{m}^{2}\right)$} \\
\hline Age adjusted & 1 & $0.74(0.65-0.83)$ & $0.60(0.53-0.67)$ & $0.55(0.49-0.62)$ & $0.48(0.41-0.55)$ & .02 \\
\hline Lifestyle adjusted & 1 & $0.81(0.71-0.91)$ & $0.76(0.67-0.86)$ & $0.74(0.65-0.85)$ & $0.70(0.60-0.81)$ & .10 \\
\hline \multicolumn{7}{|c|}{ Waist circumference $(\geq 88 \mathrm{~cm})$} \\
\hline Age adjusted & 1 & $0.66(0.50-0.87)$ & $0.51(0.39-0.67)$ & $0.48(0.37-0.63)$ & $0.46(0.35-0.61)$ & .08 \\
\hline Lifestyle adjusted & 1 & $0.74(0.55-0.98)$ & $0.64(0.48-0.85)$ & $0.65(0.49-0.86)$ & $0.67(0.50-0.96)$ & .18 \\
\hline \multicolumn{7}{|c|}{ Systolic BP $(\geq 140 \mathrm{~mm} \mathrm{Hg})$} \\
\hline Age adjusted & 1 & $0.93(0.82-1.06)$ & $0.83(0.73-0.94)$ & $0.75(0.66-0.85)$ & $0.71(0.61-0.83)$ & .21 \\
\hline Lifestyle adjusted & 1 & $0.95(0.83-1.08)$ & $0.89(0.79-1.01)$ & $0.83(0.73-0.94)$ & $0.82(0.70-0.95)$ & .22 \\
\hline \multicolumn{7}{|c|}{ Diastolic BP ( $\geq 90 \mathrm{~mm} \mathrm{Hg}$ ) } \\
\hline Age adjusted & 1 & $0.84(0.74-0.97)$ & $0.74(0.65-0.85)$ & $0.68(0.59-0.79)$ & $0.67(0.57-0.79)$ & .09 \\
\hline Lifestyle adjusted & 1 & $0.87(0.76-0.99)$ & $0.80(0.70-0.92)$ & $0.76(0.66-0.87)$ & $0.77(0.65-0.91)$ & .10 \\
\hline \multicolumn{7}{|l|}{ Heart medicine (yes) } \\
\hline Age adjusted & 1 & $0.94(0.78-1.13)$ & $0.81(0.67-0.97)$ & $0.71(0.59-0.86)$ & $0.66(0.52-0.82)$ & .14 \\
\hline Lifestyle adjusted & 1 & $0.96(0.80-1.15)$ & $0.87(0.73-1.15)$ & $0.79(0.66-0.96)$ & $0.76(0.60-0.95)$ & .15 \\
\hline
\end{tabular}

Note. Age-adjusted model: adjusted for age and year performed. Lifestyle-adjusted model: additionally adjusted for overall stress, diet habits, smoking habits, alcohol habits, level of education, relationship, current commuting, and current exercise (when not evaluated as dependent variable).

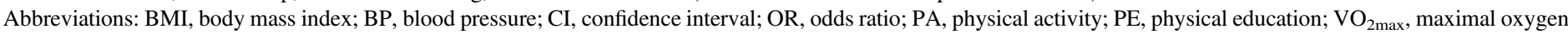
consumption.

\section{Results}

A total of 258,146 participants ( $49 \%$ women) provided data on PA prior to the age of 20 , which along with other covariates were included in the analyses. A small number of the participants (1\%) had performed the HPA before 1994, with a subsequent annual participation rate of $\geq 2000$ HPAs. For men and women in the database not included in the analyses, the majority (83\%) was due to missing data for occupation. However, comparisons of important variables between participants with missing data and those included in the analyses showed significant $(P<.001)$, but small variations (except for sex): men (59\% vs 51\%), age (43.0 vs 43.3 y), BMI (25.8 vs $\left.25.7 \mathrm{~kg} / \mathrm{m}^{2}\right)$, systolic BP (126.3 vs $126.1 \mathrm{~mm} \mathrm{Hg})$, diastolic $\mathrm{BP}(78.0$ vs $77.7 \mathrm{~mm} \mathrm{Hg}), \mathrm{VO}_{2 \max }$ (36.9 vs $36.3 \mathrm{~mL} / \mathrm{min} / \mathrm{kg}$ ), self-reported very poor/poor overall health $(6.4 \%$ vs $5.9 \%)$, very poor/poor alcohol habits $(4.9 \%$ vs $4.6 \%)$, regular exercise $(64.3 \%$ vs $64.9 \%)$, very poor/poor diet habits $(6.4 \%$ vs $8.0 \%)$, and daily smokers ( $11.1 \%$ vs $11.4 \%)$. Thus, the analyzed data can be regarded as representative for the whole HPI database population.

Characteristics of the study population are presented in Tables 1 and 2. Internal missing data are seen for $\mathrm{VO}_{2 \max }$ $[103,553$ women $(81.9 \%)$ and 107,040 men $(81.3 \%)$ provided data on $\mathrm{VO}_{2 \max }$, mainly due to participants on medication affecting heart rate response, and waist circumference [18,747 women $(14.8 \%)$ and 29,005 men $(22.0 \%)$ provided data on waist circumference], as it was an optional part of the HPA.

\section{Importance of Youth PA on Adulthood PA, Performance, and Health}

Women who participated in PE class (but with no additional PA outside school) had significantly lower OR (range: 0.66-0.84) for perceiving very poor/poor overall health, being generally and abdominal obese and having a high diastolic BP after adjusting for age and performed year of the health assessment (Table 3). In addition, they had increased OR for being current regular exercisers $(\mathrm{OR}=1.27)$ and having a normal or high $\mathrm{VO}_{2 \max }(\mathrm{OR}=1.26)$ compared with those who did not attend school PE class. These associations remained significant after adjusting for lifestyle-related covariates, albeit with a somewhat lower magnitude of ORs (range: $0.74-0.87$ and $1.17-1.23$, respectively). With more frequent participation in PA outside school hours (1-2 times/wk, 3-5 times/wk, and $\geq 6$ times/wk), even stronger beneficial associations were seen for the aforementioned outcome variables and additionally for systolic BP and the odds of not taking heart medicine. 
Table 4 OR $(95 \% \mathrm{Cl})$ for Different Levels of PA Prior to the Age of 20 in Relation to Dichotomized Variables of Perceived Health, Exercise Habits, $\mathrm{VO}_{2 \max }$, and Metabolic Health in Men

\begin{tabular}{|c|c|c|c|c|c|c|}
\hline & $\begin{array}{l}\text { No PE } \\
\text { class }\end{array}$ & Only PE class & $\begin{array}{c}\text { PE class + PA } \\
1-2 \text { times/wk }\end{array}$ & $\begin{array}{l}\text { PE class + PA } \\
3-5 \text { times/wk }\end{array}$ & $\begin{array}{c}\text { PE class }+\mathrm{PA} \\
\geq 6 \text { times/wk }\end{array}$ & $R^{2}$ \\
\hline \multicolumn{7}{|c|}{ Overall health (very poor/poor) } \\
\hline Age adjusted & 1 & $0.64(0.53-0.78)$ & $0.55(0.45-0.67)$ & $0.46(0.38-0.55)$ & $0.47(0.38-0.57)$ & .003 \\
\hline Lifestyle adjusted & 1 & $0.66(0.54-0.82)$ & $0.66(0.54-0.81)$ & $0.61(0.50-0.75)$ & $0.70(0.56-0.86)$ & .16 \\
\hline \multicolumn{7}{|c|}{ Current exercise ( $\geq 1$ time/wk) } \\
\hline Age adjusted & 1 & $1.00(0.89-1.12)$ & $1.47(1.32-1.65)$ & $2.10(1.88-2.35)$ & $2.87(2.56-3.23)$ & .04 \\
\hline Lifestyle adjusted & 1 & $0.94(0.83-1.06)$ & $1.31(1.17-1.47)$ & $1.87(1.66-2.10)$ & $2.43(2.15-2.74)$ & .13 \\
\hline \multicolumn{7}{|c|}{ Normal/high $\mathrm{VO}_{2 \max }(\geq 35 \mathrm{~mL} / \mathrm{min} / \mathrm{kg})$} \\
\hline Age adjusted & 1 & $1.10(0.96-1.26)$ & $1.54(1.34-1.76)$ & $1.97(1.72-2.25)$ & $2.47(2.15-2.84)$ & .18 \\
\hline Lifestyle adjusted & 1 & $1.09(0.95-1.26)$ & $1.34(1.16-1.54)$ & $1.59(1.38-1.83)$ & $1.80(1.56-2.08)$ & .27 \\
\hline \multicolumn{7}{|l|}{ BMI $\left(\geq 30 \mathrm{~kg} / \mathrm{m}^{2}\right)$} \\
\hline Age adjusted & 1 & $0.85(0.74-0.98)$ & $0.70(0.61-0.81)$ & $0.67(0.58-0.78)$ & $0.65(0.56-0.76)$ & .02 \\
\hline Lifestyle adjusted & 1 & $0.86(0.74-0.99)$ & $0.79(0.68-0.91)$ & $0.79(0.68-0.92)$ & $0.84(0.72-0.97)$ & .08 \\
\hline \multicolumn{7}{|c|}{ Waist circumference $(\geq 102 \mathrm{~cm})$} \\
\hline Age adjusted & 1 & $0.74(0.57-0.98)$ & $0.68(0.52-0.88)$ & $0.58(0.44-0.75)$ & $0.58(0.44-0.76)$ & .07 \\
\hline Lifestyle adjusted & 1 & $0.79(0.59-1.05)$ & $0.79(0.60-1.05)$ & $0.71(0.54-0.94)$ & $0.78(0.58-1.04)$ & .17 \\
\hline \multicolumn{7}{|c|}{ Systolic BP $(\geq 140 \mathrm{~mm} \mathrm{Hg})$} \\
\hline Age adjusted & 1 & $0.91(0.80-1.04)$ & $0.83(0.73-0.95)$ & $0.77(0.67-0.87)$ & $0.75(0.65-0.86)$ & .14 \\
\hline Lifestyle adjusted & 1 & $0.93(0.81-1.06)$ & $0.87(0.76-0.99)$ & $0.81(0.71-0.92)$ & $0.82(0.71-0.94)$ & .15 \\
\hline \multicolumn{7}{|c|}{ Diastolic BP $(\geq 90 \mathrm{~mm} \mathrm{Hg})$} \\
\hline Age adjusted & 1 & $0.89(0.77-1.02)$ & $0.78(0.68-0.90)$ & $0.74(0.65-0.86)$ & $0.76(0.65-0.88)$ & .10 \\
\hline Lifestyle adjusted & 1 & $0.90(0.78-1.04)$ & $0.83(0.72-0.96)$ & $0.81(0.70-0.93)$ & $0.86(0.74-0.99)$ & .11 \\
\hline \multicolumn{7}{|l|}{ Heart medicine (yes) } \\
\hline Age adjusted & 1 & $0.83(0.67-1.02)$ & $0.74(0.60-0.92)$ & $0.72(0.59-0.89)$ & $0.65(0.52-0.81)$ & .18 \\
\hline Lifestyle adjusted & 1 & $0.83(0.67-1.02)$ & $0.76(0.62-0.94)$ & $0.75(0.61-0.92)$ & $0.68(0.55-0.85)$ & .18 \\
\hline
\end{tabular}

Note. Age-adjusted model: adjusted for age and year performed. Lifestyle-adjusted model: additionally adjusted for overall stress, diet habits, smoking habits, alcohol habits, level of education, relationship, current commuting, and current exercise (when not evaluated as dependent variable).

Abbreviations: $\mathrm{BMI}$, body mass index; $\mathrm{BP}$, blood pressure; $\mathrm{CI}$, confidence interval; OR, odds ratio; $\mathrm{PA}$, physical activity; $\mathrm{PE}$, physical education; $\mathrm{VO}_{2 \text { max }}$, maximal oxygen consumption.

Men participating in PE class had a significant lower OR (range: 0.64-0.85) for perceiving their overall health to be very poor/poor and being both generally and abdominal obese, compared with those who did not participate in PE class (Table 4). Moreover, a more beneficial profile was seen for all the outcome variables for those with additional PA outside school hours, compared with those not participating in PE class (OR range for additional PA 1-2 times/ wk is $0.55-0.83$ for perceived and metabolic health variables, and 1.47-1.54 for current exercise and normal/high $\mathrm{VO}_{2 \max }$ ). All odds ratios, except for waist circumference, remained significant after adjusting for relevant lifestyle covariates.

In Table 5, the associations between PA prior to the age of 20 and the dichotomized outcomes were analyzed in 3 adulthood age subgroups, such as $20-35,36-50$, and $51-70$ years. Participation in PE class compared with not attending PE class was significantly important in all stages of adulthood up to 70 years for not perceiving very poor/poor overall health (range: $0.73-0.80$ ). Similar beneficial associations were seen for the odds of being a currently regular exerciser and not being obese in later adulthood, and for having a normal/high $\mathrm{VO}_{2 \max }$ in early adulthood. Participants reporting additional PA outside school hours were during all stages of adulthood more likely to exercise regularly (OR range: 1.28-1.58), have a normal/high $\mathrm{VO}_{2 \max }$ (OR range:
1.35-1.58), and not to be obese (OR range: 0.73-0.77). Similar beneficial associations, although not always significant, were seen for high waist circumference, and systolic and diastolic BP. For intake of heart medicine, beneficial associations were seen in middle and older stages of adulthood among those participating in PE class compared with those who did not.

\section{Joint Associations of Youth and Adulthood PA on Adulthood-Perceived Health and Obesity}

Compared with the reference group, those reporting not being regular exercisers in adulthood had significantly higher OR for perceiving their overall health as very poor/poor, as well as being obese regardless of youth $\mathrm{PA}$ level (PE class $+\mathrm{PA} \geq 1$ time/wk, only PE class, or no PE class; Figure 1A and 1B). However, those reporting only participating in PE class or no PE class prior to the age of 20 had higher risk of perceiving their overall health as very poor/poor and being obese, regardless of exercising or not in adulthood. Participants reporting no PE class and not being currently regularly active experienced the highest risk $(\mathrm{OR}=2.6$ for very poor/poor perceived overall health and $\mathrm{OR}=1.98$ for being obese). 
Table 5 OR $(95 \%$ Cl) for Different Levels of PA Prior to the Age of 20 in Relation to Dichotomized Variables of Perceived Health, Exercise Habits, $\mathrm{VO}_{2 \max }$, and Metabolic Health in Different Age Groups

\begin{tabular}{|c|c|c|c|c|c|c|}
\hline Age & $\begin{array}{l}\text { No PE } \\
\text { class }\end{array}$ & Only PE class & $\begin{array}{l}\text { PE class + PA } \\
1-2 \text { times/wk }\end{array}$ & $\begin{array}{l}\text { PE class + PA } \\
\text { 3-5 times/wk }\end{array}$ & $\begin{array}{c}\text { PE class + PA } \\
\geq 6 \text { times/wk }\end{array}$ & $R^{2}$ \\
\hline \multicolumn{7}{|c|}{ Overall health (very poor/poor) } \\
\hline $20-35$ & 1 & $0.75(0.57-0.99)$ & $0.73(0.56-0.96)$ & $0.70(0.54-0.92)$ & $0.70(0.52-0.93)$ & .21 \\
\hline $36-50$ & 1 & $0.80(0.65-0.98)$ & $0.79(0.65-0.97)$ & $0.77(0.63-0.94)$ & $0.96(0.77-1.19)$ & .19 \\
\hline $51-70$ & 1 & $0.73(0.58-0.92)$ & $0.70(0.56-0.87)$ & $0.68(0.54-0.86)$ & $0.70(0.54-0.91)$ & .14 \\
\hline \multicolumn{7}{|c|}{ Current exercise ( $\geq 1$ time/wk) } \\
\hline $20-35$ & 1 & $0.86(0.74-1.01)$ & $1.28(1.10-1.49)$ & $1.92(1.65-2.24)$ & $2.74(2.33-3.22)$ & .14 \\
\hline $36-50$ & 1 & $1.11(0.99-1.25)$ & $1.49(1.32-1.67)$ & $1.90(1.69-2.14)$ & $2.38(2.10-2.71)$ & .13 \\
\hline $51-70$ & 1 & $1.17(1.03-1.33)$ & $1.58(1.39-1.80)$ & $1.93(1.70-2.20)$ & $2.17(1.88-2.51)$ & .12 \\
\hline \multicolumn{7}{|c|}{$\mathrm{VO}_{2 \max }($ women $\geq 32 \mathrm{~mL} / \mathrm{min} / \mathrm{kg}$ and $\mathrm{men} \geq 35 \mathrm{~mL} / \mathrm{min} / \mathrm{kg})$} \\
\hline $20-35$ & 1 & $1.26(1.05-1.52)$ & $1.58(1.32-1.89)$ & $2.05(1.71-2.45)$ & $2.32(1.92-2.80)$ & .15 \\
\hline $36-50$ & 1 & $1.09(0.95-1.25)$ & $1.35(1.18-1.54)$ & $1.60(1.40-1.83)$ & $1.83(1.58-2.10)$ & .16 \\
\hline $51-70$ & 1 & $1.13(0.95-1.34)$ & $1.37(1.15-1.63)$ & $1.52(1.28-1.81)$ & $1.64(1.36-1.97)$ & .12 \\
\hline \multicolumn{7}{|c|}{ BMI $\left(\geq 30 \mathrm{~kg} / \mathrm{m}^{2}\right)$} \\
\hline $20-35$ & 1 & $0.87(0.71-1.07)$ & $0.73(0.59-0.89)$ & $0.64(0.52-0.78)$ & $0.59(0.47-0.73)$ & .11 \\
\hline $36-50$ & 1 & $0.86(0.74-1.00)$ & $0.78(0.68-0.91)$ & $0.79(0.68-0.92)$ & $0.83(0.71-0.97)$ & .17 \\
\hline $51-70$ & 1 & $0.79(0.68-0.93)$ & $0.77(0.66-0.90)$ & $0.80(0.68-0.94)$ & $0.87(0.73-1.04)$ & .19 \\
\hline \multicolumn{7}{|c|}{ Waist circumference (women $\geq 88 \mathrm{~cm}$ and men $\geq 102 \mathrm{~cm}$ ) } \\
\hline $20-35$ & 1 & $0.91(0.60-1.38)$ & $0.81(0.54-1.22)$ & $0.71(0.47-1.06)$ & $0.69(0.46-1.05)$ & .05 \\
\hline $36-50$ & 1 & $0.71(0.52-0.98)$ & $0.66(0.48-0.91)$ & $0.64(0.47-0.87)$ & $0.70(0.50-0.96)$ & .01 \\
\hline $51-70$ & 1 & $0.75(0.53-1.06)$ & $0.69(0.49-0.97)$ & $0.66(0.47-0.92)$ & $0.73(0.51-1.04)$ & .57 \\
\hline \multicolumn{7}{|c|}{ Systolic BP $(\geq 140 \mathrm{~mm} \mathrm{Hg})$} \\
\hline $20-35$ & 1 & $0.82(0.62-1.07)$ & $0.76(0.58-0.99)$ & $0.64(0.49-0.86)$ & $0.65(0.49-0.86)$ & .08 \\
\hline $36-50$ & 1 & $0.88(0.76-1.02)$ & $0.78(0.68-0.90)$ & $0.73(0.63-0.85)$ & $0.76(0.65-0.89)$ & .08 \\
\hline $51-70$ & 1 & $1.03(0.91-1.17)$ & $0.99(0.87-1.12)$ & $0.94(0.83-1.07)$ & $0.94(0.81-1.08)$ & .05 \\
\hline \multicolumn{7}{|c|}{ Diastolic BP ( $\geq 90 \mathrm{~mm} \mathrm{Hg}$ ) } \\
\hline $20-35$ & 1 & $0.75(0.55-1.03)$ & $0.65(0.48-0.88)$ & $0.64(0.47-0.87)$ & $0.64(0.47-0.88)$ & .06 \\
\hline $36-50$ & 1 & $0.90(0.77-1.05)$ & $0.77(0.66-0.90)$ & $0.75(0.65-0.88)$ & $0.80(0.68-0.94)$ & .07 \\
\hline $51-70$ & 1 & $0.91(0.78-1.05)$ & $0.89(0.77-1.03)$ & $0.85(0.73-0.98)$ & $0.90(0.77-1.06)$ & .03 \\
\hline \multicolumn{7}{|c|}{ Heart medicine (yes) } \\
\hline $20-35$ & 1 & $1.52(0.61-3.75)$ & $1.49(0.61-3.65)$ & $1.23(0.50-3.00)$ & $1.36(0.54-3.41)$ & .02 \\
\hline $36-50$ & 1 & $0.68(0.54-0.86)$ & $0.60(0.48-0.75)$ & $0.58(0.46-0.73)$ & $0.50(0.39-0.65)$ & .05 \\
\hline $51-70$ & 1 & $1.01(0.85-1.21)$ & $0.93(0.79-1.11)$ & $0.90(0.76-1.07)$ & $0.87(0.72-1.05)$ & .04 \\
\hline
\end{tabular}

Note. Adjusted for sex, age, performed year, overall stress, diet habits, smoking habits, alcohol habits, level of education, relationship, current commuting, and current exercise (when not evaluated as dependent variable).

Abbreviations: $\mathrm{BMI}$, body mass index; $\mathrm{BP}$, blood pressure; $\mathrm{CI}$, confidence interval; OR, odds ratio; $\mathrm{PA}$, physical activity; $\mathrm{PE}$, physical education; $\mathrm{VO}_{2 \text { max }}$, maximal oxygen consumption.

\section{Discussion}

The present results show that participation in school-based PE class, compared with not participating, was associated with more beneficial perceived health, a more active life, higher $\mathrm{VO}_{2 \max }$, and a better metabolic health in adulthood in a sample of over 250,000 working Swedish men and women. Additional PA outside school hours is associated with even stronger beneficial associations in both men and women for all studied outcome variables. These associations are maintained even after adjusting for important confounders. Further, in analyses of the impact of age (up to $70 \mathrm{y}$ ) and hence time since leaving school, the associations were similar, with some variations between stages of adulthood. To our knowledge, no previous study has included a population-based sample of this size to retrospectively evaluate the associations between PE class participation and PA levels in childhood/adolescence to adulthood PA level, $\mathrm{VO}_{2 \max }$, and health up to 70 years of age.

The beneficial associations found for those participating in minimum PE class compared with those not participating in PE class are highly important. In women, PE class participation was associated with better current perceived health, regular exercise habits, higher $\mathrm{VO}_{2 \max }$, lower levels of overall and abdominal obesity, and lower diastolic BP. In men, similar associations were seen for perceived health, and overall and abdominal obesity. Although the goal and content of the education plan for the PE class have been reformed and revised throughout the years, one of the most common aims of PE in many countries (including Sweden) 

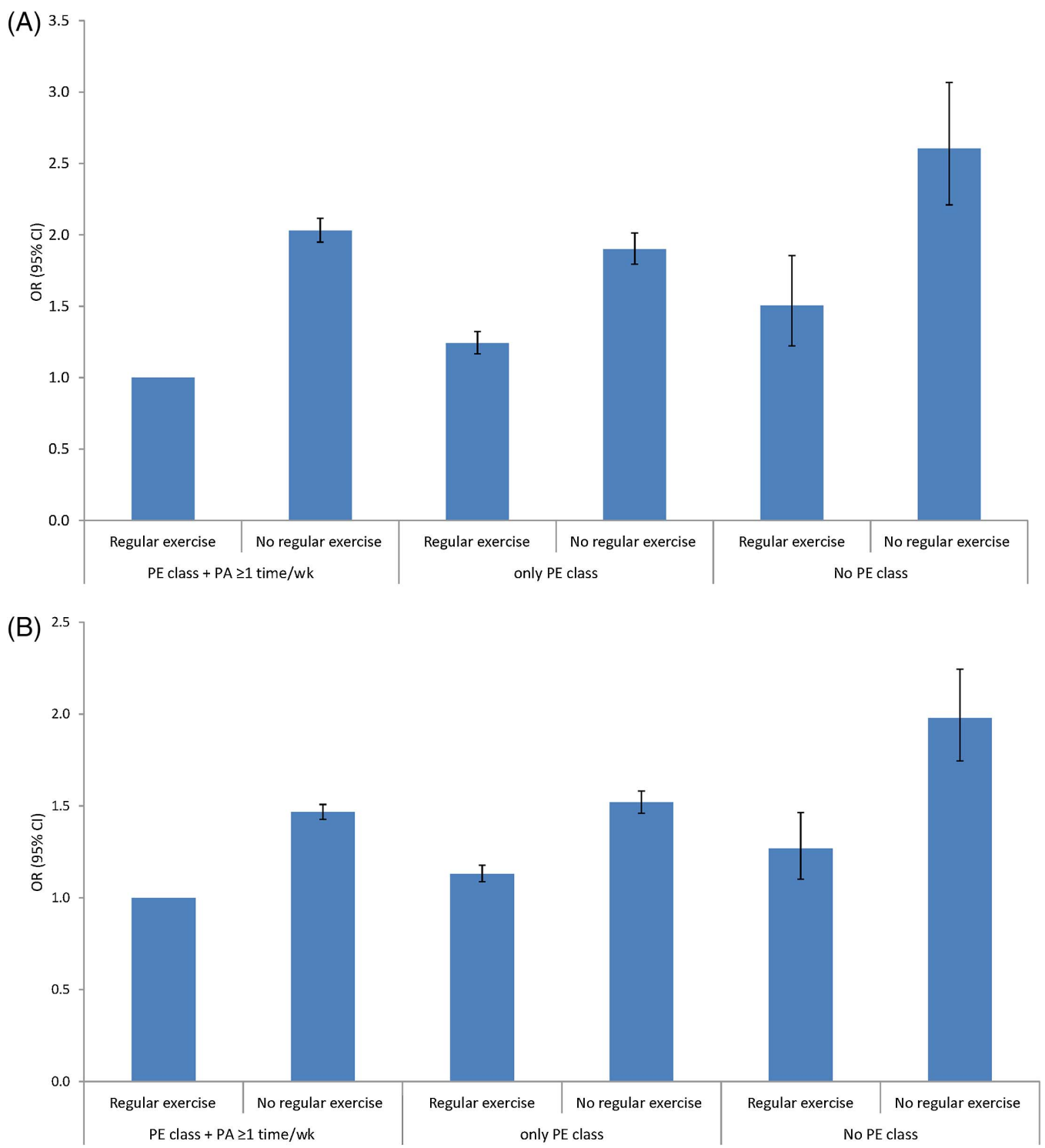

Figure 1 - (A) OR (95\% CI) for perceived poor health in relation to cross-tabulation of previous PA (prior to 20 years of age) and current exercise habits. OR (95\% CI) was for each group from left to right: 1 (ref.), 2.03 (1.94-2.12), 1.24 (1.17-1.32), 1.90 (1.79-2.01), 1.51 (1.22-1.86), and 2.60 (2.21$\left.3.07 ; R^{2}=.18\right)$. (B) OR $(95 \% \mathrm{CI})$ for BMI $\geq 30 \mathrm{~kg} / \mathrm{m}^{2}$ in relation to cross-tabulation of previous PA (prior to 20 years of age) and current exercise habits. OR $(95 \% \mathrm{CI})$ was for each group from left to right: 1 (ref.), 1.47 (1.43-1.51), 1.13 (1.09-1.18), 1.52 (1.46-1.58), 1.27 (1.10-1.46), and 1.98 (1.74-2.24; $R^{2}=.09$ ). Adjusted for sex, age, performed year, overall stress, diet habits, smoking habits, alcohol habits, level of education, relationship, and current commuting. PE indicates physical education; PA, physical activity; OR, odds ratio; CI, confidence interval; ref., reference; BMI, body mass index.

is to promote a lifelong physically active lifestyle. ${ }^{18}$ In addition, skill development and habits in childhood are of great importance for higher exercise habits in adult life. ${ }^{19}$ Hence, the present results indicating that PE class participation alone increases the possibility to be active in adulthood is especially important for those individuals who are not engaged in additional PA outside of school hours, as school PE classes are thought to present a diversity of activities for the child/adolescent to try and perform.

PE class participation (in women) and, in a dose-response manner, increasing PA outside school hours increase significantly the odds of exercise regularly, as well as having a higher $\mathrm{VO}_{2 \max }$ later in life for both men and women. This is an important finding and in line with previous research. Cleland et $\mathrm{al}^{9}$ reported significant associations with total weekly PA at follow-up (mean age: 26-36 y) in boys aged 9-12 and girls aged 13-15 at baseline; however, they reported that compulsory school PE (in minutes per week) in the same population showed no relationships. ${ }^{10}$ In a retrospective study, Kraut et $\mathrm{al}^{20}$ reported that those participating in organized school sports had a more than 3-fold increased odds of being weekly regularly active after 40 years of age. Hirvensalo et $\mathrm{al}^{21}$ reported a more than 2 -fold increased odds for being regularly weekly active on at least moderate intensity at 70 years of age, if reporting participation in competitive sport prior to 20 years of age. In general, tracking of PA from adolescence to older adulthood is in general reported to be low to medium with more nonsignificant associations (indicating poorer stability) in women compared with men. ${ }^{22}$ This might be due to a low number of participants in some studies, shorter follow-up period, or both.

The finding of $30 \%$ lower OR for poor/very poor perceived overall health among men and women reporting minimum PE class 
participation, compared with nonparticipants, is highly interesting. Poor perceived health, assessed through a simple question as in the present study, has in large population-based samples been identified as a single, strong predictor of all-cause mortality. ${ }^{23,24}$ Importantly, the present associations remained even after adjustments for other factors associated with poor health, such as low level of education, bad diet habits, and smoking, and are strong and evident in all stages of adulthood (Table 5). Interestingly, the further reduced risk of poor overall health with additional PA outside school hours in the fully adjusted analyses is small. This may point out that nonparticipants in school PE classes belong to a risk group for adult-impaired health and other outcomes, but it might also indicate that even low levels of PA engagement prior to the age of 20 are important for future overall health.

We found strong associations with lower overall and abdominal obesity in both men and women in those with only PE class participation and increasingly with additional PA hours outside school. A similar association was seen for diastolic BP in women. For both systolic BP and heart medicine intake, significant associations were present in those reporting at least 1 hour of PA outside school. Previous research on the effects of youth PA on cardiovascular disease risk (CVD) in adulthood is limited. Rangul et al ${ }^{14}$ investigated differences in PA patterns between adolescence (13- to 19-y-old boys and girls) and young adulthood (23-31 y) for cardiometabolic risk. They found that those who maintained their PA from adolescence to adulthood had a significantly lower CVD and better mental health, compared with those who remained inactive. This may be comparable to the results in Figure 1A and $1 \mathrm{~B}$ in the present study. Those who did not attend PE class and did not exercise regularly in adulthood had a 2-fold increased OR for overall obesity and 2.6-fold increased OR for poor health compared with those who had maintained an active lifestyle from adolescence to adulthood. Importantly, participants in the present study who adopted an active lifestyle from adolescence to adulthood had a significantly lower OR for both poor perceived health and overall obesity (Figure 1A and 1B). This is evident in those with no PE class as well as only PE class during adolescence and indicates the importance of present activity levels for metabolic health and well-being. On the contrary, participants who adopted an inactive lifestyle (participating in only PE class or PE class + additional PA hours outside school during adolescence, but with no regular exercise in adulthood) had significantly lower OR for both poor perceived health and overall obesity compared with those being inactive maintainers from adolescence to adulthood. This illustrates the possible importance of PA habits in early life, although not continuing being active, which evidently influences health and well-being through other pathways.

It should be noted that it is not possible to draw any definite conclusions regarding causality in these associations between youth PA and different adult outcomes, because many transitions and life-changing events occur during the course of life. Moreover, we lack information on the reasons for not participating in PE class for the participants. Although being a compulsory subject in the Swedish school system since the 19th century, there are students who choose to not participate. In a study of 1000 high-school students in southern Sweden, 1 of 4 did not attend at least one-third of PE class opportunities. ${ }^{25}$ Few students had medical reasons for not participating, with psychological (PE class perceived as boring/ meaningless, bad self-confidence, less satisfied with own athletic ability, physical appearance, and health) and social (peer pressure to not participate and bullying) reasons being more frequently reported. In addition, the PE class having a competitive approach where students need to perform well and achieve sporting results, together with being directed at and including mainly interested students, ${ }^{26,27}$ may serve as unmotivating factors for students equivocal to the subject and increase the risk of dropping out. Swedish junior high-school and high-school students identified as frequent dropouts from PE class were found to smoke and drink alcohol more often, skip other compulsory classes, have a higher BMI, enjoy school less, and have less interest in school work, compared with other students attending PE class on regular basis. ${ }^{28}$ Girls dropping out from PE class were also more often bullied. Only $14 \%$ reported some physical or psychological hinderance that could have prevented participation. External risk factors for PE class dropout were mandatory showering after PE class, grades depending on physical performance, far-off located sports facilities, and PE class taking place in the early morning or at lunch time. Girls more often than boys have been reported to experience unease and discomfort for PE class, ${ }^{26}$ which may influence participation. In the present study, more women than men reported not participating in PE class. However, it is important to emphasize that those participants reporting not attending school PE classes, regardless of the reason, must be considered as belonging to a risk group for adult physical inactivity, low $\mathrm{VO}_{2 \max }$, and poor perceived overall health, as well as measured impaired metabolic health later in life.

\section{Strength and Limitations}

The strength of this study is the large number of men and women studied with standardized methods. Another strength is also the duration during which current data were obtained, making variations in perceived health and other factors not depending on variations in changes in the society. A novelty is the long positive tracking effects of childhood/adolescence PA on adult health up to 70 years, hence providing a possibility to study the associations at different stages of adulthood. A limitation is that we have no information on why participants did not participate in PE class. In addition, there is no information regarding level and amount of PA in school PE class, but modern research regarding nonintentional physical exercise has shown that even walking and other lowintensity nonsport activities have positive effects on health and longevity. ${ }^{29}$ Health problems in youth may influence adult $\mathrm{VO}_{2 \max }$ and PA. Moreover, we do not have any information regarding intensity and amount of PA outside school hours, or information regarding whether PE was taught by PE specialists, at what dosage and at which level of education. Another limitation could be that retrospective information on PE class participation and PA outside school hours may suffer from recall bias. However, PE class participation might be a more definite decision, which may be easier to recall than hours per week of PA. However, any uncertainty of additional PA hours outside school should be equal in all PA classes. Although the data collection was not initially intended for research purposes, the standardization of procedures and quality control is well suited for such analyses.

\section{Conclusions}

PE class participation, compared with not participating, was associated with more beneficial perceived health, a more active life, higher $\mathrm{VO}_{2 \max }$, and a better metabolic health in adulthood in a sample of over 250,000 working Swedish men and women. Additional PA outside school hours induced even stronger associations. When analyzing the impact of age up to 70, and hence 
time since leaving school, the associations were similar, with some small variations between stages of adulthood. Joint analyses revealed that both youth PA and current PA were important for reducing the probability of having poor health and being obese in adulthood. For those not participating in leisure-time PA, PE class may provide an important opportunity to engage in and try a diversity of activities, which may promote a lifelong active and healthy lifestyle. It also seems essential to encourage previously inactive individuals to engage in PA, as adulthood PA level regardless of youth PA, to decrease probability of poor health and obesity.

\section{Acknowledgments}

The authors gratefully acknowledge the Health Profile Coaches from all over Sweden. A special thanks to the members of staff at HPI Health Profile Institute AB. No external funding received.

\section{References}

1. Ekblom-Bak E, Olsson G, Ekblom O, Ekblom B, Bergstrom G, Borjesson $\mathrm{M}$. The daily movement pattern and fulfilment of physical activity recommendations in Swedish middle-aged adults: the SCAPIS pilot study. PLoS ONE. 2015;10(5):e0126336. PubMed doi:10. 1371/journal.pone.0126336

2. Hagstromer M, Troiano RP, Sjostrom M, Berrigan D. Levels and patterns of objectively assessed physical activity-a comparison between Sweden and the United States. Am J Epidemiol. 2010; 171(10):1055-1064. PubMed doi:10.1093/aje/kwq069

3. Paffenbarger RS Jr, Wing AL, Hyde RT. Physical activity as an index of heart attack risk in college alumni. Am J Epidemiol. 1978;108(3): 161-175. PubMed doi:10.1093/oxfordjournals.aje.a112608

4. Byberg L, Melhus H, Gedeborg R, et al. Total mortality after changes in leisure time physical activity in 50 year old men: 35 year follow-up of population based cohort. Br Med J. 2009;338:b688. doi:10.1136/ bmj.b688

5. Karlsson MK, Nordqvist A, Karlsson C. Physical activity increases bone mass during growth. Food Nutr Res. 2008;52:1871. doi:10 .3402/fnr.v52i0.1871

6. Kosti RI, Panagiotakos DB. The epidemic of obesity in children and adolescents in the world. Cent Eur J Public Health. 2006;14(4): 151-159. PubMed

7. Ekblom O, Oddsson K, Ekblom B. Health-related fitness in Swedish adolescents between 1987 and 2001. Acta Paediatr. 2004;93(5): 681-686. PubMed doi:10.1111/j.1651-2227.2004.tb02997.x

8. Tammelin T, Nayha S, Hills AP, Jarvelin MR. Adolescent participation in sports and adult physical activity. Am J Prev Med. 2003; 24(1):22-28. PubMed doi:10.1016/S0749-3797(02)00575-5

9. Cleland V, Dwyer T, Venn A. Which domains of childhood physical activity predict physical activity in adulthood? A 20-year prospective tracking study. Br J Sports Med. 2012;46(8):595-602. PubMed doi:10.1136/bjsports-2011-090508

10. Cleland V, Dwyer T, Blizzard L, Venn A. The provision of compulsory school physical activity: associations with physical activity, fitness and overweight in childhood and twenty years later. Int J Behav Nutr Phys Act. 2008;5:14. PubMed doi:10.1186/1479-5868-5-14

11. Huotari PR, Mikkelsson L, Kujala UM, Aakso LL, Nupponen H. Physical activity and fitness in adolescence as predictors of selfestimated fitness in adulthood. J Sports Med Phys Fitness. 2013; 53(2):177-184. PubMed

12. Telama R, Yang X, Leskinen E, et al. Tracking of physical activity from early childhood through youth into adulthood. Med Sci Sports
Exerc. 2014;46(5):955-962. PubMed doi:10.1249/MSS.0000000000 000181

13. Jose KA, Blizzard L, Dwyer T, McKercher C, Venn AJ. Childhood and adolescent predictors of leisure time physical activity during the transition from adolescence to adulthood: a population based cohort study. Int J Behav Nutr Phys Act. 2011;8:54. PubMed doi:10.1186/ 1479-5868-8-54

14. Rangul V, Bauman A, Holmen TL, Midthjell K. Is physical activity maintenance from adolescence to young adulthood associated with reduced CVD risk factors, improved mental health and satisfaction with life: the HUNT study, Norway. Int J Behav Nutr Phys Act. 2012;9:144. PubMed doi:10.1186/1479-5868-9-144

15. Andersson G. The Importance of Exercise for Sick Leave and Perceived Health. [PhD dissertation]. Sweden: Department of Preventive and Social Medicine, Linköping University; 1987.

16. Lilliecreutz Huitema E, Andersson G, Samuelsson K. Lifestyle changes with help from Health Profile Assessment in combination with support in individual interventions for persons with acquired brain injury-a pilot study. Eur J Physiother. 2014;16:151-158.

17. Åstrand I. Aerobic work capacity in men and women with special reference to age. Acta Physiol Scand Suppl. 1960;49(169):1-92. PubMed

18. Phüse U, Gerber M. International Comparison of Physical Education: Concepts, Problems, Prospects. Oxford, UK: Meyer \& Meyer Sport; 2005.

19. Kuh DJ, Cooper C. Physical activity at 36 years: patterns and childhood predictors in a longitudinal study. J Epidemiol Community Health. 1992;46(2):114-119. PubMed doi:10.1136/jech.46.2.114

20. Kraut A, Melamed S, Gofer D, Froom P, Study C. Effect of school age sports on leisure time physical activity in adults: the CORDIS study. Med Sci Sports Exerc. 2003;35(12):2038-2042. PubMed doi:10. 1249/01.MSS.0000099087.96549.96

21. Hirvensalo M, Lintunen T, Rantanen T. The continuity of physical activity - a retrospective and prospective study among older people. Scand J Med Sci Sports. 2000;10(1):37-41. PubMed doi:10.1034/ j.1600-0838.2000.010001037.x

22. Telama R. Tracking of physical activity from childhood to adulthood: a review. Obes Facts. 2009;2(3):187-195. PubMed doi:10.1159/ 000222244

23. Ganna A, Ingelsson E. 5 year mortality predictors in 498, 103 UK Biobank participants: a prospective population-based study. Lancet. 2015;386(9993):533-540. PubMed doi:10.1016/S0140-6736(15) 60175-1

24. DeSalvo KB, Bloser N, Reynolds K, He J, Muntner P. Mortality prediction with a single general self-rated health question. A metaanalysis. J Gen Intern Med. 2006;21(3):267-275. PubMed doi: 10.1111/j.1525-1497.2005.00291.x

25. Larsson L. Idrott och hälsa är ingenting för mig [Physical Education and Health is Nothing for Me]. [Master thesis]. Stockholm, Sweden: Swedish School of Sport and Health Sciences; 2003.

26. Redelius K. Bäst eller pest? Ämnet idrott och hälsa bland elever i grundskolans senare år [Best or pest? The subject of physical education and sport among pupils in the later years of compulsory school]. In: Larsson H, Redelius K, eds. Mellan nytta och nöje. Bilder av ämnet idrott och hälsa [Between Use and Pleasure. Images of the Subject Physical Education]. Stockholm, Sweden: Swedish School of Sport and Health Sciences; 2004:149-172.

27. Sandahl B. Ett ämne för alla?: normer och praktik i grundskolans idrottsundervisning 1962-2002 [A Subject for Everyone?: norms and Practice of Compulsory Physical Education in Swedish Schools 1962-2002]. [PhD thesis]. Stockholm, Sweden: Swedish School of Sport and Health Sciences; 2005. 
28. Bråkenhielm G. Ingen gympa för mig! - en undersökning av skälen till att elever inte deltar i ämnet idrott och hälsa [No Physical Education for Me!-A Study on the Reasons to Not Participate in Physical Education]. Stockholm, Sweden: Svensk Idrottsforskning; 2008:2.
29. Ekblom-Bak E, Ekblom B, Vikstrom M, de Faire U, Hellenius ML. The importance of non-exercise physical activity for cardiovascular health and longevity. Br J Sports Med. 2014;48(3):233-238. PubMed doi:10.1136/bjsports-2012-092038 